\title{
Familial amyotrophic lateral sclerosis with a point mutation of SOD-1: intrafamilial heterogeneity of disease duration associated with neurofibrillary tangles
}

Richard W Orrell, Andrew W King, David A Hilton, Malcolm J Campbell, Russell J M Lane, Jacqueline S de Belleroche

\begin{abstract}
Mutations of SOD-1 have recently been associated with autosomal dominant familial amyotrophic lateral sclerosis (ALS). A patient is described with a 20 year duration of motor neuron disease, with clinical features of ALS, who was heterozygous for a point mutation ATT to ACT leading to substitution of isoleucine for threonine at codon 113 in exon 4 of SOD-1. This mutation has previously been described in two families with ALS and three apparently sporadic cases of ALS. The patient described here had a family history suggestive of autosomal dominant inheritance of this genetic mutation; other members of the family having a more typical disease duration. Unusual pathological features included neurofibrillary tangles in neurons of the globus pallidus, substantia nigra, locus coeruleus, and inferior olivary nuclei, and absence of ubiquitin immunoreactive inclusions in motor neurons. This may reflect the slow progression of the neurodegeneration associated with the SOD1 mutation in this patient. The prolonged survival, of over 20 years, with other family members having a more typical survival of two to three years, has important implications for genetic counselling in families with ALS in addition to the fundamental biological questions concerning the influence of these mutations on disease expression.
\end{abstract}

(F Neurol Neurosurg Psychiatry 1995;59:266-270)

Keywords: amyotrophic lateral sclerosis; familial ALS; SOD-1; neurofibrillary tangles

Around $5-10 \%$ of patients with amyotrophic lateral sclerosis (ALS) (or motor neuron disease) have a family history of the disease, and in larger families show evidence of autosomal dominant inheritance. Genetic studies in such large families led to the identification of linkage to chromosome 21 in around $40 \%$ of families. ${ }^{1}$ This linkage was recently attributed to mutations in the SOD-1 gene which encodes the enzyme copper-zinc superoxide dismutase (SOD). ${ }^{2}$ Reduced SOD enzyme activity has been identified in erythrocytes and other tissues in these patients, ${ }^{34}$ although it is possible that the disease expression is due to a toxic effect of the mutant enzyme. ${ }^{5}$ To date, around 25 different missense mutations of SOD-1 with single base pair substitutions, ${ }^{6}$ and a single family with a two base pair deletion, ${ }^{7}$ have been described. Such mutations are present in around $20 \%$ of all families with familial ALS in North America. The clinical implications of these mutations are of great importance. We describe a family with a SOD-1 mutation, in whom the considerable variability of disease expression illustrates the potential difficulties in prognosis and genetic counselling that may be encountered.

\section{Patients and methods}

CASE HISTORY

This woman first noticed tripping and difficulty climbing stairs at the age of 48 . At the age of 49 she developed weakness of the right thumb, with difficulty grasping a car door or keys. At that time there was pronounced wasting of the right thenar eminence and interossei of the right hand, with severe weakness. There was also weakness of the right triceps. Reflexes were abnormally brisk in both arms, but examination was otherwise normal. An EMG showed features of active denervation of both hands and the right leg, with fasciculations in the right tibialis anterior and right thenar eminence, and normal motor and sensory conduction.

By the age of 60 , she had progressive wasting and weakness, being only able to walk 100 yards, with elbow crutches. There was some weakness in both hands. She had slight difficulty with tongue movement. By the age of 63 the course of the disease had accelerated; she was confined to a wheelchair, with leg, trunk, and distal arm weakness. She still had proximal limb function and was able to feed herself. There was spasticity of the upper limbs. She had wasting and weakness of the face, mouth and tongue, with dysarthria. There were fasciculations in the tongue and a brisk jaw jerk, but no clinical signs of extrapyramidal disease or dementia. She died of pneumonia at the age of 69 .

PEDIGREE (FIG 1)

The proband is IV.13. One brother (IV.12) was killed in action in the 1914-18 war; the other brother (IV.10) is aged 80, and has no neurological disease. The proband's mother (III.8) died at the age of 50 with a diagnosis of "amyotrophic lateral sclerosis of the spine" 
Figure 1 Pedigree of represent males, circles females, diamonds further generations of between one and three members. Oblique lines represent symbols represent those with $A L S$, the partially filled symbol possible ALS. The proband is indicated by an arrow. family with $A L S$. Squares deceased members. Filled

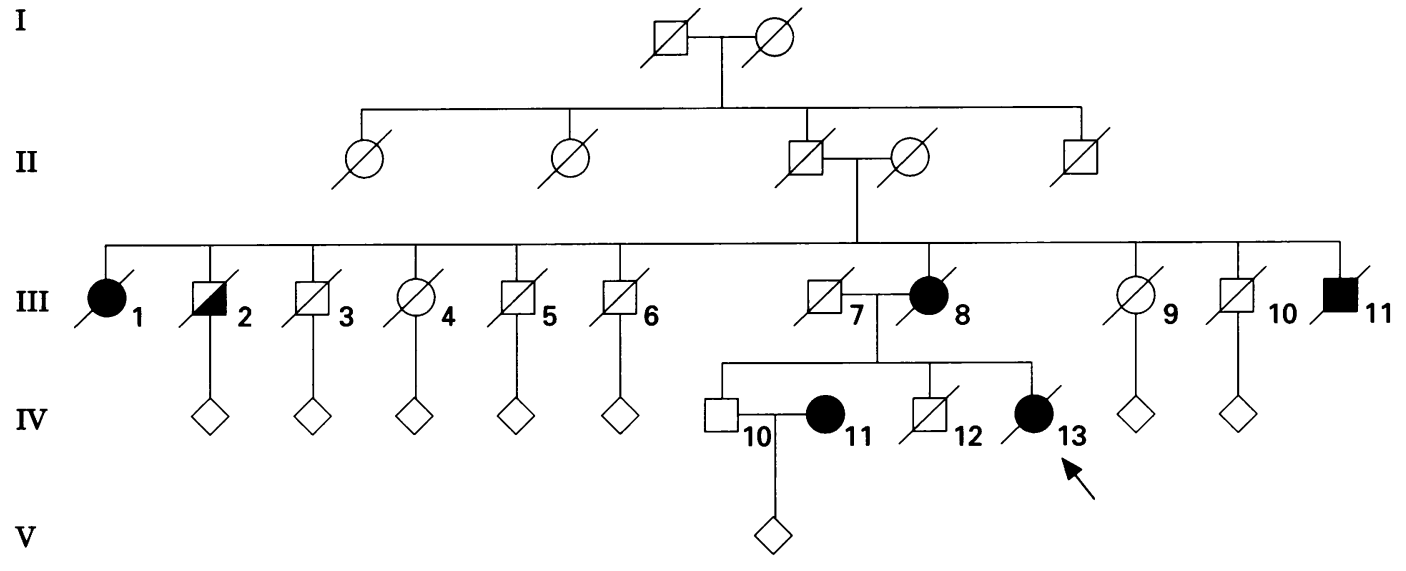

and a disease duration of between two and three years. The family was large and contact has been lost among all the relatives, but the following descriptions are given.

III. 1 This woman died at the age of about 50 , in 1930, of ALS with a disease duration of between four and five years.

III. 2 This man had a full life span, although there is a family rumour that he died of ALS, developing when aged 65.

III.3 This man died with no evidence of ALS.

III.4 This woman died of heart disease in her 70 s.

III.5 This man was killed in action in the 1914-18 war.

III.6 This man died in his 70 s of heart disease.

III.9 This woman died aged 89 of pneumonia.

III.10 This man died of old age.

III.11 This man developed mild leg weakness at the age of 70 , presenting with weakness of the right arm followed by the left arm at the age of 72. This progressed to generalised weakness, wasting, and fasciculations of all limbs and tongue, with brisk reflexes and extensor plantar responses. He died of ALS, with a disease duration of around three years.

\section{Results}

NEUROPATHOLOGICAL FINDINGS

Before fixation the brain weighed $1360 \mathrm{~g}$. The fixed brain appeared macroscopically unremarkable. The spinal cord showed obvious

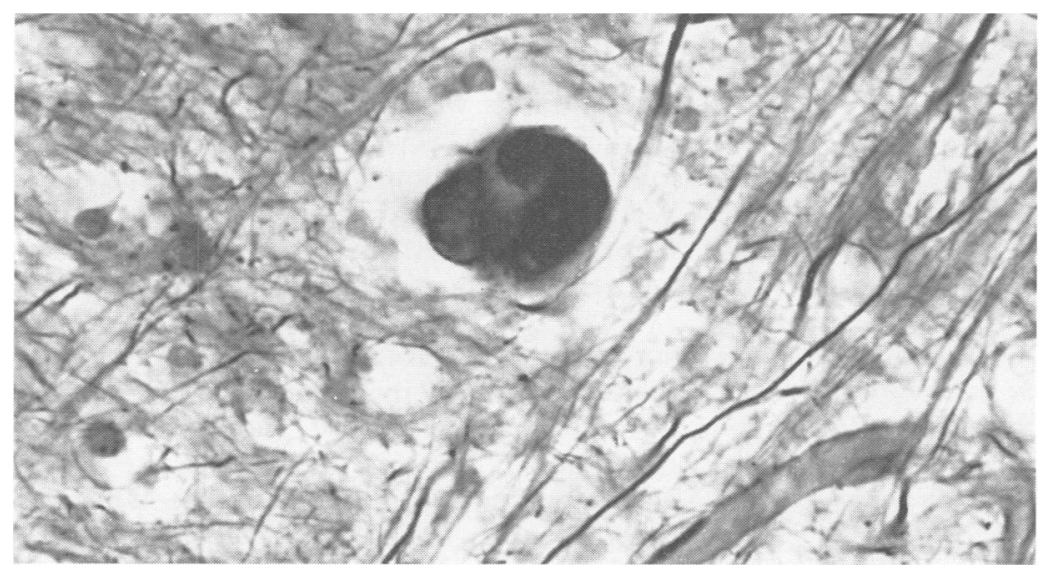

Figure 2 Neuron of globus pallidus, with intact nucleus, showing a neurofibrillary tangle. Palmgren's; $\times 380$. atrophy of the anterior nerve roots, most pronounced in the mid-cervical region.

\section{Skeletal muscle}

Paraffin sections showed many atrophic angulated muscle fibres, some scattered singly within fascicles, others in relatively large groups. The appearances were typical of chronic partial denervation.

\section{Spinal cord}

Histological examination disclosed widespread loss of anterior horn cells and Clarke's column nuclei, with preservation of Onuf's nucleus. The anterior spinal nerve roots were depleted of fibres and contained excessive endoneurial collagen. Occasional axonal spheroids were present in the anterior horn regions of the lumbar cord. The posterior roots appeared normal. Pronounced fibre loss was noted in both anterior and posterior spinocerebellar tracts on myelin staining, with additional loss of myelin staining in the corticospinal tracts. The posterior columns appeared normal. No neurofibrillary tangles or other neuronal inclusions were seen in sections of spinal cord stained with haematoxylin and eosin or modified Bielschowsky silver impregnation.

\section{Brain}

Sections of the frontal and temporal lobes were unremarkable, and in particular there were no Alzheimer type changes. The globus pallidus showed diffuse gliosis, neuronal loss, and scattered neurofibrillary tangles (fig 2). These tangles were argyrophilic but did not fluoresce under ultraviolet light after thioflavin-S staining. No tangles were seen in the putamen or caudate nuclei, which appeared normal.

Mild neuronal loss, pigmentary incontinence, and neurofibrillary tangles were also present in the substantia nigra and locus coeruleus. Within the medulla there was neuronal loss, gliosis, and occasional neurofibrillary tangles in the inferior olivary nuclei. Neuronal loss was also seen in the seventh and 12th cranial nerve nuclei.

\section{Immunocytochemistry}

Sections of temporal lobe, basal ganglia, midbrain, and pons were immunostained by a 
conventional avidin-biotin-horseradish peroxidase method. Controls included sections from cases of Alzheimer's disease and sections from which the primary antibodies were omitted during immunostaining. Occasional neurofibrillary tangles reacted with BF10 (a monoclonal antibody to phosphorylated medium weight polypeptide of neurofilament). Antibodies to ubiquitin (Dako) and RT-97 (a monoclonal antibody to phosphorylated heavy

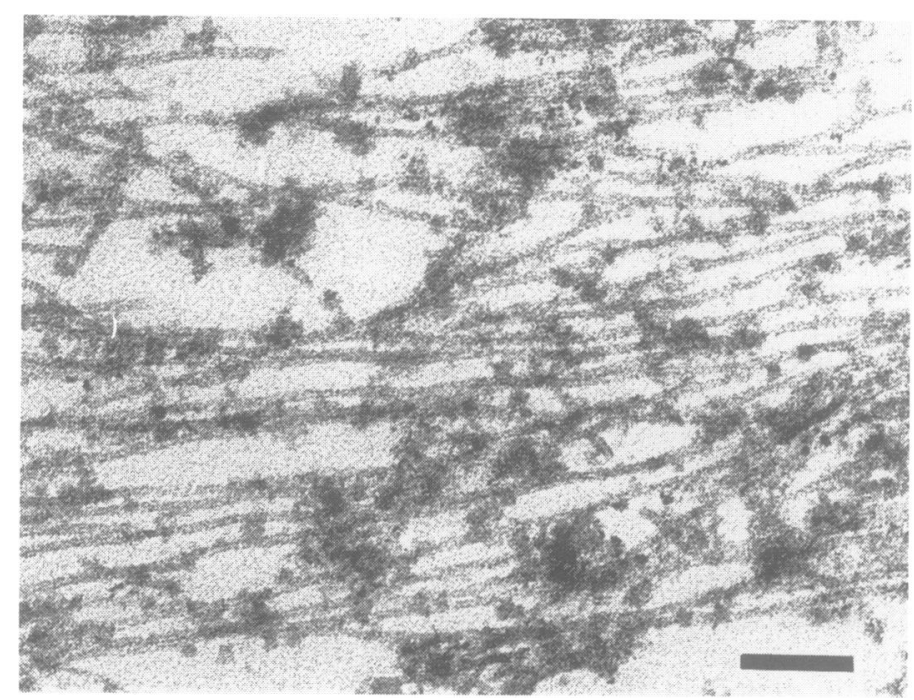

Figure 3 Electron micrograph showing an intraneuronal neurofibrillary tangle in the substantia nigra composed of straight $8 \mathrm{~nm}$ filaments; bar $=100 \mathrm{~nm}$.

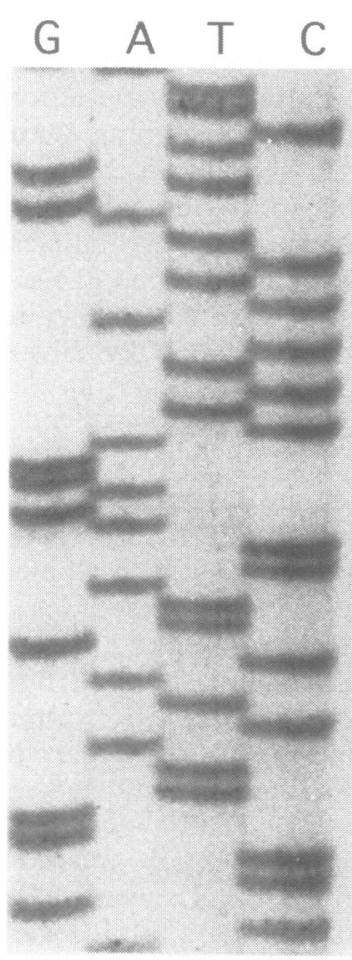

a

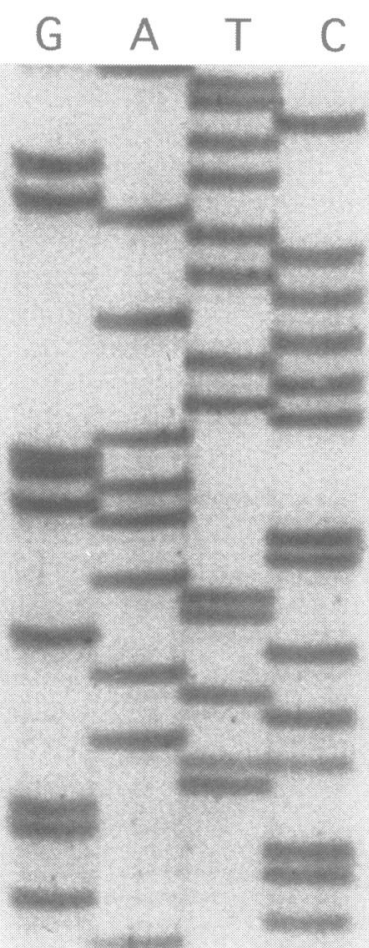

b
Figure 4 The sequence of exon 4 in a normal subject (a) and the proband (b). The four lanes for each individual represent the nucleotides guanine $(G)$, adenine $(A)$, thymine $(T)$, and cytosine $(C)$. These have been separated electrophoretically on a polyacrylamide gel, and the sequence of the nucleotides, which represents the "genetic code" of the DNA, is read sequentially from top to bottom. The nucleotides have separated according to their distance from the primer, giving the code T TCT GTG ATC etc. An additional C band (arrowed) is present in the proband (b) which is not present in the normal subject (a). In the proband (b), the mutant $C$ band and its corresponding normal $T$ band, are both of reduced intensity as this is a heterozygote point mutation $T$ to $C$. weight neurofilament) gave negative results. Occasional tangles showed weak tau protein (Sigma) immunoreactivity. Moderate numbers of tau positive neurites were present in the globus pallidus and dentate nuclei, but were only occasionally seen in the substantia nigra, oculomotor nuclei, and basis pontis. tau positive astrocytes were not noted.

Immunohistochemistry of multiple sections of spinal cord did not show any ubiquitinated inclusions, but showed diffuse immunoreactivity in the perikaryon of several anterior horn cells with BF10 neurofilament antibody.

\section{Electron microscopy}

Ultrastructural examination of sections of substantia nigra showed neurofibrillary tangles composed of bundles of straight filaments approximately $8 \mathrm{~nm}$ in diameter (fig 3).

SOD-1 SEQUENCE ANALYSIS

DNA was extracted from whole blood with Nucleon II (Scotlab). Each of the five exons of SOD-1 was amplified with appropriate primers. The primers for exon 4 were 5'CGCGACTAACAATCAAAGTGA and 5'CATCAGCCCTAATCCATCTGA. A polymerase chain reaction (PCR) was performed with $1 \mu \mathrm{g}$ of genomic DNA in a $100 \mu \mathrm{l}$ reaction volume with $5 \mu \mathrm{l}$ DMSO, $1.5 \mathrm{mM}$ $\mathrm{MgCl}_{2}, 10 \mu \mathrm{l} 10 \times \mathrm{PCR}$ buffer IV (Advanced Biotechnologies) $(10 \times$ buffer IV contains $200 \mathrm{mM}\left(\mathrm{NH}_{4}\right)_{2} \mathrm{SO}_{4}, 750 \mathrm{mM}$ Tris $\mathrm{HCl}(\mathrm{pH}$ 9.0), $\quad 0.1 \% \quad$ Tween), $\quad 200 \mu \mathrm{M}$ dNTPs (Advanced Biotechnologies), $2 \cdot 5$ units Taq polymerase, $0.5 \mathrm{pM}$ of biotinylated and nonbiotinylated primers. The reaction mixture was overlaid with mineral oil before thermal cycling (Hybaid Omnigene thermal cycler), at $95^{\circ} \mathrm{C}$ for two minutes, 32 cycles of annealing at $55^{\circ} \mathrm{C}$ for two minutes, extension at $72^{\circ} \mathrm{C}$ for two minutes, strand separation at $95^{\circ} \mathrm{C}$ for one minute, and a final extension at $72^{\circ} \mathrm{C}$ for six minutes. The products were screened by electrophoresis in $2 \%$ agar stained with ethidium bromide, the product size being 232 base pairs. The amplified product was separated on Dynabeads M-280 Streptavadin (Dynal, Norway) by the standard protocol, and the single stranded product was directly sequenced using a Sequenase 7 Deaza dGTP sequencing kit with Sequenase version $2.0 \mathrm{~T} 7$ DNA polymerase (United States Biochemical Corporation) labelled with ${ }^{35}$ SdATP (Amersham), according to the protocol. The DNA strands were electrophoresed on a $6 \%$ acrylamide gel (Severn Biotech Ltd) at $60 \mathrm{~W}$ for one to two hours, the gel transferred to Watman paper, dried, and exposed to Hyperfilm (Amersham) for up to seven days before developing and reading the sequence.

\section{SOD-1 MUTATION IDENTIFICATION}

A heterozygote point mutation was identified in the proband in exon 4, with the change of the codon ATT to ACT, causing the amino acid substitution Ile113Thr (fig 4). This was not present in the patient's clinically normal brother or in control specimens examined. There was no mutation in exons $1,2,3$, and 5 . 
The mutation was confirmed by sequencing the complementary strand.

\section{Discussion}

SOD-1 MUTATION

Twenty five missense mutations of SOD-1 have now been described in familial ALS. The point mutation in exon $4, \mathrm{~T}$ to $\mathrm{C}$, causing the amino acid substitution Ile113Thr, has been reported in two families with $\mathrm{ALS}^{2}$ and in three patients with apparently sporadic ALS in Scotland. ${ }^{8}$ The isoleucine at position 113 of SOD is highly conserved among species, including drosophila, swordfish, mouse, and human. ${ }^{3}$ It is structurally located within the Greek key loop, and forms twofold symmetric dimer interactions. A substitution should significantly destabilise the mutant heterodimeric and homodimeric SOD. The mechanism by which these enzymic changes cause disease is yet to be defined, but may be by free radical damage due to reduced enzyme activity, ${ }^{9}$ or other neurotoxic mechanisms due to a different function of the altered enzyme. ${ }^{5}$ The clinical detail in these reports is sparse.

\section{PATHOLOGY}

An unusual feature of this case is the finding of neurofibrillary tangles in neurons of the globus pallidus, substantia nigra, locus coeruleus, and inferior olivary nuclei. The ultrastructural and immunocytochemical findings suggest that the tangles are composed of neurofilaments. Neurofibrillary tangles are not usually seen in either sporadic or familial ALS, ${ }^{10}$ although they are well recognised in Guamanian ALS ${ }^{11}$ and have been described in cases of ALS occurring as a delayed complication of encephalitis lethargica. ${ }^{12} 13$ The neurofibrillary tangles in Guamanian ALS have a more widespread distribution from those in the present case, also occurring in the cerebral cortex, dentate nucleus, and spinal cord, ${ }^{11}$ having a paired helical structure ${ }^{14}$ and exhibiting strong immunoreactivity with antibodies to tau protein. ${ }^{15}$ Although Guamanian ALS may be related to environmental factors, recent segregation analyses support the involvement of a major gene in combination with environmental factors. ${ }^{16}$ No mutation of SOD-1 was identified, however, in eight Guam patients with ALS and six patients with parkinsonism, some of whom had other affected family members. ${ }^{17}$ Neurofibrillary tangles are a prominent feature of progressive supranuclear palsy, but the tangles in the patient we describe are much fewer than normally seen in progressive supranuclear palsy. The patient had clear clinical features of ALS, and not of progressive supranuclear palsy, although progressive supranuclear palsy may occasionally be familial. ${ }^{18}$

Although neurofibrillary tangles are not common in either sporadic or familial ALS, other cytoskeletal abnormalities often occur. These include various neuronal inclusions ${ }^{19}$ and abnormal accumulations of neurofilaments within neuronal somata and axons, ${ }^{20-22}$ with focal, rather than diffuse, neurofilamen- tous accumulations being more common in familial cases. $^{23}$ Ubiquitin immunoreactive inclusions are a common finding in neurodegenerative diseases, including motor neurons of the anterior horn, brain stem, and, rarely, the cortex in ALS. ${ }^{24}$ These inclusions are particularly associated with an aggressive and short duration of disease. ${ }^{25}$ The absence of ubiquitinated inclusions in this patient may reflect the slow progression of the disease. This would support the hypotheses that ubiquitin immunoreactive inclusions result from the accumulation of altered cytoskeletal protein during relatively short duration, intense or sustained cell stress saturating ubiquitin mediated proteolytic clearance, ${ }^{26}$ but not saturating the system in more slowly progressive disease. Alternatively, ubiquitination is not a primary cause of the neurodegeneration. The neuropathological findings in the current case may represent part of the range of neurofibrillary tangle formation described in cases of familial ALS. ${ }^{27-30}$ Alterations in neurofilament density can cause slowing of axonal transport $^{31}$ and, conversely, toxic agents which interfere with axonal transport are associated with neurofilament accumulation. ${ }^{32-34}$ The relation between mutations in the SOD-1 gene and abnormal neurofilament accumulation in ALS remains uncertain, although such mutations may lead to structural change in the SOD protein and interference with axoplasmic flow. Neurofilaments have been shown to accumulate in animal models of motor neuron disease ${ }^{35}$ and, recently, transgenic animals have been described in which overexpression of neurofilament subunits by neurons ${ }^{36}{ }^{37}$ or a point mutation in the neurofilament subunit, ${ }^{38}$ have resulted in disorders resembling motor neuron disease.

\section{CLINICAL GENETICS}

The SOD-1 mutation segregates with the disease in an autosomal dominant manner, and can also be found in asymptomatic members of a family. ${ }^{39}$ The age dependent and incomplete penetrance of the gene is acknowledged. ${ }^{539}$ An additional complication in the counselling of people with the SOD-1 mutation is the variability of disease duration and severity. ${ }^{40}$ If the proband presented now at age 48 years with a SOD-1 mutation, the advice given would have to be very general to anticipate her survival of over 20 years, living alone, and requiring a nursing home only after 17 years duration of disease at the age of 65 . It would not be appropriate to take into account the rapid (two to three years) duration of disease of the other members of the family. Generally, a later onset of disease may be associated with a more rapid course, ${ }^{41}$ but in itself cannot account for the variation in this family.

In Japan a mutation of SOD-1 was reported to be associated with a relatively benign disease..$^{42}$ In the members of these two families the mean survival after onset was 17 (SD 11) years. These statistics were based on only six patients, whereas a total of 23 were shown to be affected on the pedigrees. ${ }^{42}$ 
They suggested that this represents a new subtype, or mild form, of ALS associated with a unique mutation (His46Arg). We suggest that the prolonged survival data may be an artefact of the longest surviving being available for clinical and genetic analysis. The $95 \%$ confidence intervals of their data clearly include the more rapid (two to three years) survival, and counselling of a relatively benign disease for all those in such families may be inappropriate.

It is possible that this variable expression in a large family, including a relatively benign form of ALS, explains the appearance of apparently sporadic forms in smaller families with the same mutation. It is thus possible that a proportion of apparently sporadic forms, especially in smaller modern families, may be due to inheritance of SOD-1 mutations. It is important to determine the biological basis of the variable age of onset and identify other contributory factors to fully understand the causes and treatment of ALS.

This work was supported by grants from the Motor Neurone Disease Association and the Special Trustees of Charing Cross and Westminster Medical School. We acknowledge the use of the resources of the MRC human genome mapping project. Neurofilament antibodies BF10 and RT-97 were a kind gift from Professor BH Anderton, Institute of Psychiatry, London.

1 Siddique T, Figlewicz D, Pericak-Vance MA, et al. Linkage of a gene causing familial amyotrophic lateral sclerosis to chromosome 21 and evidence of genetic heterogeneity. N Engl f Med 1991;324:1381-4.

2 Rosen DR, Siddique T, Patterson D, et al. Mutations in $\mathrm{Cu} / \mathrm{Zn}$ superoxide dismutase gene are associated with familial amyotrophic lateral sclerosis. Nature 1993;362: familial

3 Deng H, Hentati A, Tainer J, et al. Amyotrophic lateral sclerosis and structural defects in $\mathrm{Cu}, \mathrm{Zn}$ superoxide dismutase. Science 1993:261:1047-51.

4 Bowling AC, Schulz JB, Brown RH, Beal MF. Superoxide dismutase activity, oxidative damage, and mitochondrial energy metabolism in familial and sporadic amyotrophic lateral sclerosis. $\mathcal{F}$ Neurochem 1993;61:2322-5.

5 Gurney ME, Haifeng P, Chiu A. Motor neuron degeneration in mice that express a human $\mathrm{Cu}, \mathrm{Zn}$ superoxide dismutase mutation. Science 1994;264:1772-5.

6 Orrell RW, deBelleroche JS. Superoxide dismutase and amyotrophic lateral sclerosis. Lancet 1994;344:1651-2.

7 Pramatarova A, Goto J, Nanba E, et al. A two base pair deletion in the SOD-1 gene causes familial amyotrophic lateral sclerosis. Human Molecular Genetics 1994;3: 2061-2.

8 Jones CT, Brock DJH, Chancellor AM, Warlow CP, Swingler RJ. Cu/Zn superoxide dismutase (SOD1) mutations and sporadic amyotrophic lateral sclerosis. Lancet 1993;342:1050-1.

9 Olanow CW. A radical hypothesis for neurodegeneration. TINS 1993;16:439-44

10 Hudson AJ. Amyotrophic lateral sclerosis and its association with dementia, parkinsonism and other neurological disorders: a review. Brain 1981;104:217-47.

11 Hirano A, Malamud N, Elizan TS, Kurland LT. Amyotrophic lateral sclerosis and Parkinsonismdementia complex on Guam. Further pathologic studies. Arch Neurol 1966;15:35-51.

12 Hirano A, Zimmerman HM. Alzheimer's neurofibrillary changes. A topographic study. Arch Neurol 1962;7: $227-42$.

13 McMenemy WH, Barnard RO, Jellinek EH. Spinal amyotrophy. A late sequel of epidemic encephalitis (von Economo). Revue Roumaine de Neurology 1967;4:251-9.

14 Guiroy DC, Miyazaki M, Multhaup G, et al. Amyloid of neurofibrillary tangles of Guamanian parkinsonismdementia and Alzheimer disease share an identical amino acid sequence. Proc Natl Acad Sci USA 1987; 84:2073-7.

15 Shankar SK, Yanagihara R, Garruto RM, et al. Immunocytochemical characterisation of neurofibrillary tangles in amyotrophic lateral sclerosis and parkinsonism-dementia of Guam. Ann Neurol 1989;25:146-51.

16 Bailey-Wilson JE, Plato CC, Elston RC, Garruto RM. Potential role of an additive genetic component in the cause of amyotrophic lateral sclerosis and parkinsonismdementia in the Western Pacific. Am 7 Med Genet 1993;45:68-76.

17 Figlewicz DA, Garruto RM, Krizus A, Yanagihara R, Rouleau GA. The $\mathrm{Cu} / \mathrm{Zn}$ superoxide dismutase gene in ALS and parkinsonism-dementia of Guam. Neuro Report ALS and parkin

18 Brown J, Lantos P, Stratton M, Roques P, Rossor M Familial progressive supranuclear palsy. $f$ Neurol Neurosurg Psychiatry 1993;56:473-6.

19 Lowe J. New pathological findings in amyotrophic lateral sclerosis. I Neurol Sci 1994;124(suppl):38-51.

20 Carpenter S. Proximal axonal enlargement in motor neuron disease. Neurology 1968;18:841-51.

21 Hirano A, Donnenfeld H, Schoichi S, Nakano I. Fine structural observations of neurofilamentous changes in amyotrophic lateral sclerosis. $¥$ Neuropathol Exp Neurol 1984;43:461-70.

22 Chou SM. Pathology: light microscopy of amyotrophic lateral sclerosis. In: Smith RA, ed. Handbook of amyotrophic lateral sclerosis. New York: Marcel Dekker, 1992:503-17.

23 Mizusawa H, Matsumoto S, Yen SH, Hirano A, RojasCorona RR, Donnenfeld H. Focal accumulation of phosphorylated neurofilaments within anterior horn cells phosphorylated neurofilaments within anterior horn cells
in familial amyotrophic lateral sclerosis. Acta Neuropathol in familial amyotrophic

24 Leigh PN, Garofalo O. The molecular pathology of motor neuron disease. In: Leigh PN, Swash M, eds. Motor neuron disease. London: Springer-Verlag, 1995:139-61.

25 Schiffer D, Autilio-Gambetti L, Chio A, et al. Ubiquitin in motor neuron disease: study at the light and electron
microscope. $\mathcal{F}$ Neuropathol Exp Neurol 1991;50:463-73.

26 Manetto V, Perry G, Tabaton M, et al. Ubiquitin is associated with abnormal cytoplasmic filaments characteristic of neurodegenerative diseases. Proc Natl Acad Sci USA of neurodegenerativ

27 Finlayson MH, Guberman A, Martin JB. Cerebral lesions in familial amyotrophic lateral sclerosis and dementia. in familial amyotrophic lateral sclerosis

28 Schmitt HP, Emser W, Heimes C. Familial occurrence of amyotrophic lateral sclerosis, parkinsonism, and dementia. Ann Neurol 1984;16:642-8.

29 Kato S, Hirano A. Involvement of the brain stem reticular formation in familial amyotrophic lateral sclerosis. Clin Neuropathol 1992;11:41-4.

30 Takahashi $\mathrm{H}$, Oyanagi $\mathrm{K}$, Ikuta $\mathrm{F}$, Tanaka $M$, Yuasa $\mathrm{T}$, Miyatake T. Widespread multiple system degeneration in a patient with familial amyotrophic lateral sclerosis. Neurol Sci 1993;120:15-21.

31 de Waegh SM, Lee VMY, Brady ST. Local modulation of neurofilament phosphorylation, axonal caliber and slow axonal transport by myelinating Schwann cells. Cell axonal transport

32 Griffin JW, Hoffman PN, Clark AW, Carroll PT, Price DL. Slow axonal transport of neurofilament proteins: impairment by $\beta, \beta^{\prime}$-iminodipropionitrile administration. Science 1978;202:633-5.

33 Graham DG, Szakal-Quin G, Priest JW, Anthony DC. In vitro evidence that covalent crosslinking of neurofilaments occurs in gamma-diketone neuropathy. Proc Nat Acad Sci USA 1984;81:4979-82.

34 Troncoso JC, Hoffman PN, Griffin JW, Hess-Kozlow KM, Price DL. Aluminium intoxication: a disorder of neurofilament transport in motoneurons. Brain Res 1985;342:172-5.

35 Cork LC, Griffin JW, Choy C, Padula CA, Price DI. Pathology of motor neurons in accelerated hereditary
canine spinal muscular atrophy. Lab Invest 1982;46: 89-99.

36 Cote F, Collard JF, Julien JP. Progressive neuronopathy in transgenic mice expressing the human neurofilament heavy gene: A mouse model of amyotrophic lateral sclerosis. Cell 1993;73:35-46.

37 Xu Z, Cork LC, Griffin JW, Cleveland DW. Increased expression of neurofilament subunit NF-L produces morphological alterations that resemble the pathology of human motor neuron disease. Cell 1993;73:23-33.

38 Lee MK, Marszalek JR, Cleveland DW. A mutant neurofilament causes massive, selective motor neuron death implications for the pathogenesis of human motor neuron disease. Neuron 1994;13:975-88.

39 Robberecht W, Sapp P, Viane MK, et al. Cu/Zn superoxide dismutase activity in familial and sporadic amyotrophic lateral sclerosis. $¥$ Neurochem 1994;62:384-7.

40 Appelbaum IS, Roos RP, Salazar-Grueso EF, et al. Intrafamilial heterogeneity in hereditary motor neuron Intrafamilial heterogeneity in hereditar

41 Norris F, Shepherd R, Denys E, et al. Onset, natural history and outcome in idiopathic adult motor neuron history and outcome in idiopathic a

42 Ogasawara M, Matsubara Y, Narisawa $\mathrm{K}$, et al. Mild ALS in Japan associated with novel SOD mutation. Nature Genetics 1993;5:323-4.

43 Aoki M, Ogasawara M, Matsubara Y, et al. Familial amyotrophic lateral sclerosis (AIS) in Japan associated with H46R mutation in $\mathrm{Cu} / \mathrm{Zn}$ superoxide dismutase gene: possible new subtype of familial ALS. $\exists$ Neurol $S c i$ 1994;126:77-83. 